\title{
GREY-OUT ELIMINATION: THE ROLES OF SPATIAL WAVEFORM, FREQUENCY AND PHASE
}

\author{
Rachel Corfield, J. P. Frosdick and F. W. Campbell \\ The Physiological Laboratory. Downing Street, Cambridge. CB2 3EG. U.K.
}

(Received 20 June 1977; in revised form 4 January 1978)

\begin{abstract}
The retinal events of a saccadic eye movement were simulated by presenting to the stationary eye a blank field of variable duration bracketed in time by vertical gratings of the same average space luminance. The blank represents the saccadic "grey-out" and the gratings represent the clear retinal images of each fixational pause.

The blank field fails to be be perceived when it is of short duration. This duration is dependent on the waveform and spatial frequency of the gratings and may be as long as $350 \mathrm{msec}$.

The effect is termed "grey-out elimination" and could explain our failure to perceive the retinal smear produced by saccades.
\end{abstract}

\section{INTRODUCTION}

Campbell and Wurtz (1978) have shown that there is little or no loss of visual acuity during saccadic eye movements and yet in normal situations we never see the smeared retinal image which occurs during every saccade. They have termed this very low contrast smear which is a consequence of the rapid movement of an image over the retina the saccadic "greyout".

In the experiments reported here the retinal events of a saccade are simulated by presenting to the stationary eyes a blank field of variable duration bracketed in time by a vertical grating of the same mean luminance. The blank field represents the saccadic grey-out, the grating represents the clear patterned retinal image of each fixational pause.

During an actual saccade the whole visual field must grey-out. In the simplified simulation forced upon us by technical limitations only the oscilloscope screen greys out to the space average luminance of the gratings. To minimize the possible effects of the rectangular borders of the oscilloscope it was surrounded by a further screen matched in colour and luminance to the oscilloscope display. The display subtended $20^{\circ}(\mathrm{V})$ by $25^{\circ}(\mathrm{H})$ and the surround $52^{\circ}$ (V) by $78^{\circ}(\mathrm{H})$; thus there was a reasonable simulation of saccadic grey-out in the greater part of the visual field.

Substantial durations of the blank field fail to be perceived when preceded and followed by a patterned field in this way. This type of perceptual elimination may well be analagous to the situation during saccadic eye movements and these experiments were designed to define and quantify the parameters of the eliminating stimuli.

Footnote. One of the referees of this manuscript has pointed out that the briefer duration at which the perception of a blank is eliminated with the in-phase first and third harmonics (Table 1 ) is analagous to the lessened adaptive effect on threshold of the two sinusoids as compared to the adpative effect of either alone, as shown by Stecher. Sigel and Lange (1973).
We do not wish to imply any active inhibition of visual function by the gratings, but merely to refer to phenomenological failure to perceive the blank. The neutral term "elimination" has therefore been coined.

\section{METHODS}

The subjects in these experiments sat in a lighted room facing an oscilloscope screen on which vertical gratings of various waveforms. and independently variable spatial frequency and contrast. could be generated. The mean luminance of the screen remained constant at all times at $100 \mathrm{~cd} / \mathrm{m}^{2}$ as measured on an SEI photometer.

In all experiments the display on the oscilloscope screen took the form of a uniform field (blank), alternating with a grating. Care was taken to ensure that the change from a blank to a grating involved no alteration of average space luminance over the screen. The screen surround was a large piece of white card illuminated to be of equal colour and brightness to the screen. The pattern dimensions were $25^{\circ}(\mathrm{H})$ by $20^{\circ}(\mathrm{V})$ in most of the experiments. One temporal cycle, consisting of one presentation of the blank followed by one of the gratings, had a constant duration of $1 \mathrm{sec}$. The duration of the blank could be varied. relative to that of the grating, by the subject turning a potentiometer.

The subjects in the majority of the experiments were the authors RC and JPF. RC and JF, who acted as subject in Expt 2, both wore a spectacle correction. The acuity of all three subjects was better than $6 / 6$ (Snellen notation) in both eyes.

\section{RESULTS}

The subjects' task in all experiments was to adjust the duration of the blank period until they could just no longer see it as a separate event (i.e. method of adjustment). This duration was measured on a digital counter, and was termed "critical-blank-duration". The settings of critical-blank-duration made by the subject indicate the power of the gratings preceding and following the blank to eliminate its perception. Longer critical-blank-durations indicate greater eliminative power.

Subjects were not provided with a fixation point 
but were instructed to "look steadily" at the centre of the screen. If the subjects were aware of making a blink or a large saccade immediately before, during or after any blank whilst making a setting of criticalblank-duration they ignored that particular observatron. This was an infrequent occurrence and the small standard errors of the settings indicate a lack of contamination by this artifact.

It is important to note that during the blank period the screen was evenly illuminated rather than dark and thus, in going from grating to blank. no mean luminance change was involved. If a luminance change did occur during the blank period subjects saw this as a slight, brief dimmer or brighter pulse, although they still failed to see the blank itself as long as its duration was shorter than the criticalblank-duration. This is analagous to the situation during real saccades where any changes in mean luminance that occur are usually small, except in cases where the saccade traverses a high contrast object, such as a bright window. In the latter case the mean luminance change may be noticed as a brief bright pulse although the window itself will not be seen.

For durations of blank equal to or shorter than the critical-blank-duration, although subjects did not perceive the blank as a separate event they were aware that something had occurred. This took the form of an apparent movement of the grating. For high frequency gratings the movement was smooth and the grating appeared to shift by 1 cycle or more across the screen to right or left. For low frequency gratings, however, the grating appeared to move in a small jerk.

As the blank duration became significantly less than the critical-blank-duration the apparent movement of all gratings became less marked and more jerky.

\section{EXPERIMENT :}

Dependence of Critical-Blank-Duration on Spatial Frequency

In these initial experiments the contrast of the sinewave gratings was kept constant at $30 \%$ throughout, while their spatial frequency was varied from trial to trial. The blank period on any trial was preceded and followed by gratings identical in contrast, spatial frequency and phase.

The screen subtended $26^{\circ}$ by $20^{\circ}$ during the majortty of trials; however, in order to increase the available range of spatial frequencies it was necessary, on some trials, to double the subjects' distance from the screen, thus reducing the field size. Field size did not seem to be a determining factor of critical-blankduration.

In any one trial, at a given spatial frequency, the subject was allowed unlimited time in which to make a single setting of critical-blank-duration. Spatial frequency was varied in a random manner between trials and at least ten settings of critical-blank-duration were made at each spatial frequency.

The results (Fig. 1) indicate that critical-blankduration increases with increasing spatial frequency. The relationship between critical-blank-duration and $\log$ spatial frequency appears to be linear. The best

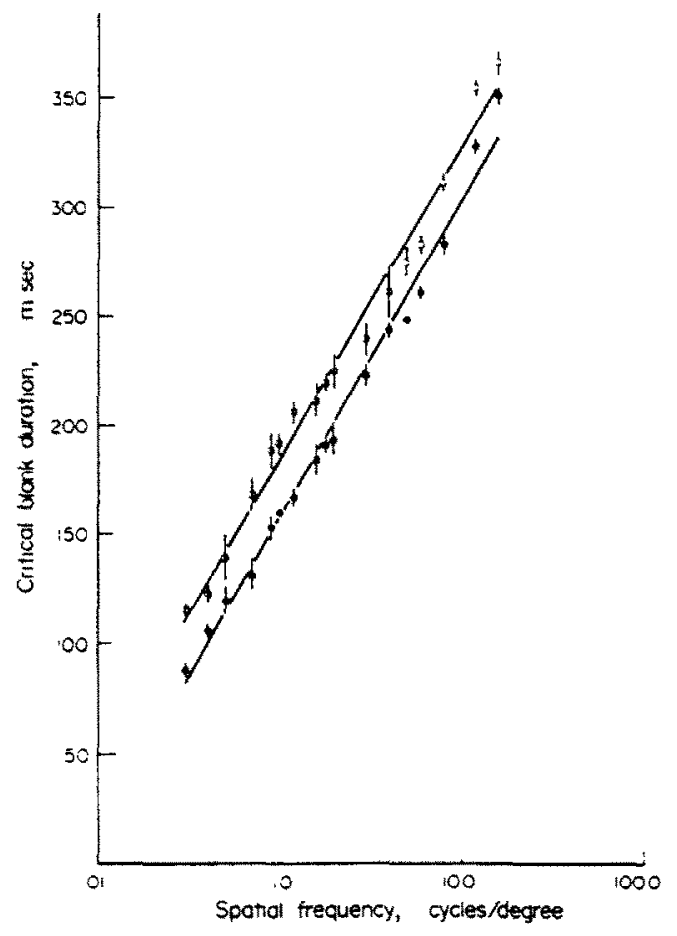

Fig. 1. Dependence of critucal-blank-duration on spatual frequency. Open circles show data for JPF, filled circles show data for RC. Spatial frequency is plotted along the abscissa on a logarithmic scale. Vertical bars at each data point represent $\pm 2 S$.E. The two straight lines represent the best linear fit to the data by least-squares method. Regression coefficients are higher than 0.99 for both lines and their slopes are not significantly different.

straight line through the data for each subject (by least-squares method) is shown in Fig. 1. The correlation coefficient is better than 0.99 for both sets of data. This logarithmic plot gave higher correlation when tested than did any other simple relationship. The lines for the two subjects are parallel; the slopes of the regression lines are not significantly different ( $t$-test). However, settings of critical-blank-duration for JPF are 20-30 msec longer at all spatial frequencies than those for RC. This difference between the subjects was maintained throughout the entire series of experiments.

\section{EXPERIMENT 2}

\section{Dependence of Critical-Blank-Duration on Contrast}

Having shown the dependence of critical-blankduration on spatial frequency. the effect of varying the contrast of the grating at a fixed spatial frequency was investigated.

Subjects made settings of critical-blank-duration as before. A range of contrasts between 3 and $74 \%$ was investigated at two different spatial frequencies $(0.5$ and $2.0 \mathrm{c} / \mathrm{deg}$ ). The spatial frequency and contrast of the gratings were varied randomly between trials. Ten settings of critical-blank-duration were made at each contrast for both spatial frequencies.

The results (Figs $2 A$ and $B$ ) clearly demonstrate the lack of dependence of critical-blank-duration on contrast; at both spatial frequencies the variation of 


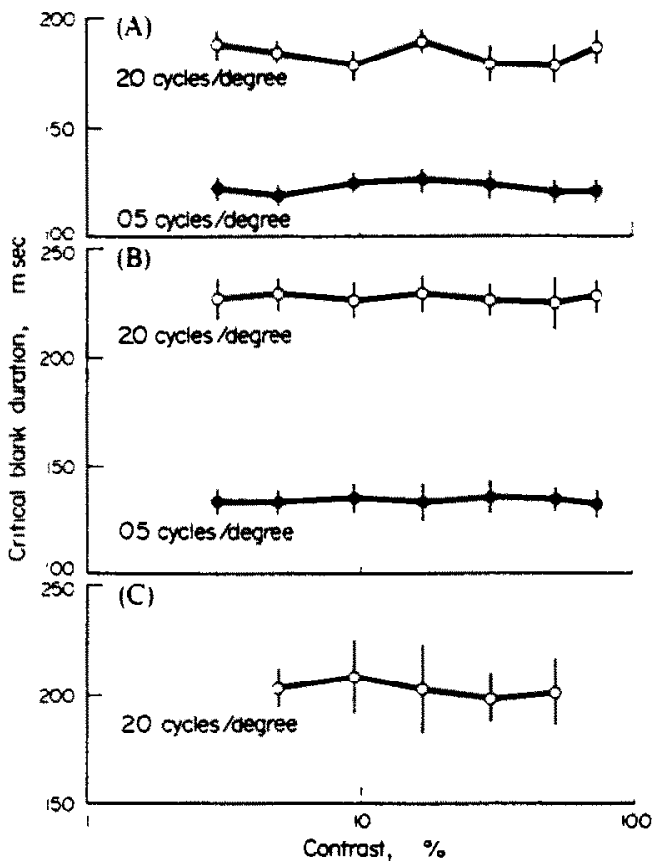

Fig. 2. Effect of contrast on critical-blank-duration. Data for subjects RC. JPF and JF (naive) are shown in Figs 2A. B and C. respectively. Vertical bars at each data point represent \pm 2 S.E. Contrast is plotted on a logarithmic scale.

critical-blank-duration with contrast is not statistically significant.

The critical-blank-durations obtained for the two spatial frequencies used in this experiment agree well with those values for the corresponding spatial frequencies in Expt 1, and the difference between subjects' settings was again observed.

This result was so surprising that a confirmatory experiment was carried out to test a totally naive subject who could have no preconception of the result. Figure $2 \mathrm{C}$ shows the data for this subject. JF confirms the independence of critical-blank-duration and contrast. The larger standard deviations in the results reflect this subjects' relative lack of experience in the task.

\section{EXPERIMENT 3}

\section{Properties of Square-Wave Gratings}

An investigation into the eliminative properties of square-wave gratings, considered in terms of their Fourier components, was then made.

Since square-waves contain spatial frequencies higher than their fundamental frequency (i.e. $3 r d$, 5 th, 7 th harmonics, etc.) it would be expected that a square-wave would give a longer critical-blankduration than a sine-wave of the same fundamental frequency. We have already shown that for higher spatial frequencies eliminative power is greater. Figure 3 shows that this assumption is incorrect. The data shows settings of critical-blank-duration made by RC for both square-wave and sine-wave gratings. All data of Fig. 3 were obtained in a single experimental run, varying spatial frequency and waveform from trial to trial. The experiment was repeated for JPF and the data showed the same trends.

Thus, square-waves have less power than sinewaves to eliminate perception of the blank. the critical-blank-duration being consistently lower for the square-wave at all spatial frequencies tested As the curve approaches values of spatial frequency where sine- and square-waves are indistinguishable (because the higher harmonics are not perceived), the difference in critical-blank-duration between sine- and squarewaves is reduced, as shown in the figure.

\section{EXPERIMENT 4}

\section{Properties of Complex Waveforms}

In order to further investigate the rather unexpected result of the previous experiment other complex waveforms were looked at. Five main waveforms of grating were used and each was investigated at three different fundamental frequencies $10.33,0.66$ and $1.5 \mathrm{c} / \mathrm{deg}$ ).

The five waveforms were as follows:

(1) A sine-wave of the fundamental frequency.

(2) A square-wave of the fundamental frequency.

(3) A sine-wave of three times this frequency and one third the amplitude of the fundamental (third harmonic).

(4) A combination of 1 and 3 (fundamental plus third harmonic) added in-phase (peak-subtract mode).

(5) A combination of 1 and 3 added $180^{\circ}$ out-ofphase (peak-add mode) (See Table 1).

The waveform presented was varied at random between trials, the subject making 20 settings of critical-blank-duration for each of the five waveforms. A

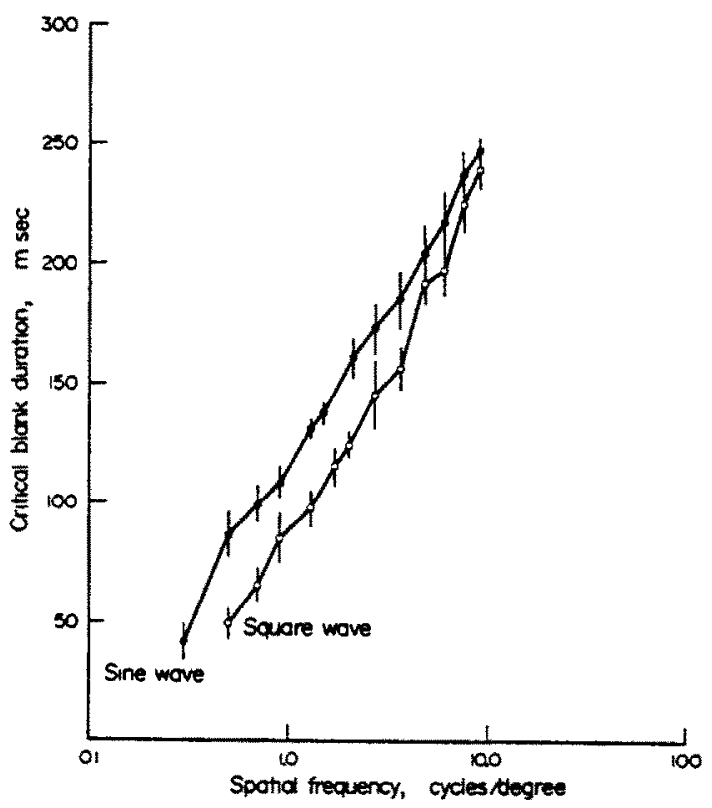

Fig. 3. Comparison of eliminative properties of squareand sine-wave gratings. The data for $R C$ and JPF gave similar results. Vertical bars at each data point represent \pm 2 S.E. Spatial frequency is plotted on a logarithmic scale as before. 
Table | Elrminative properties of complex waveforms

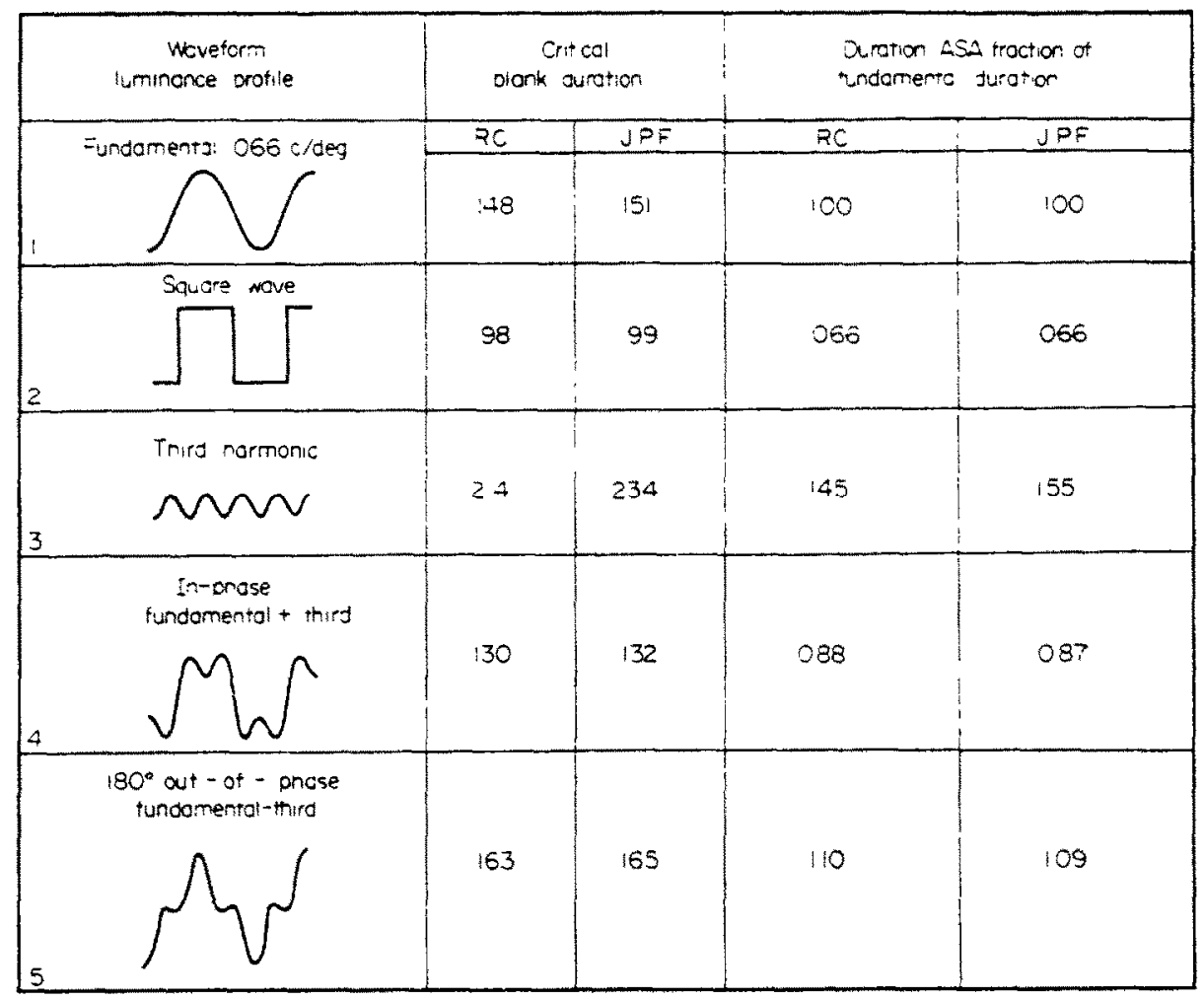

Luminance profiles of complex uaveforms used are shown in the left-hand column. Right-hand columns give tatio of crittcal-blank-duration for each complex waveform to that of the fundamental.

whole run was carried out at any one fundamental frequency before progressing to the next. Table I gives results at fundamental frequency $0.66 \mathrm{c} / \mathrm{deg}$ : results for the two other fundamental frequencies were similar.

Elimination of perception of the blank by the third harmonic alone proved to be better than for the fundamental (longer critical-blank-duration). This was to be expected. due to its higher spatial frequency (Expt 1) and despite its lower amplitude. eliminative power being independent of contrast (Expt 2). Data for the square-wave also confirmed the earlier resuit (Expt 3 ). critical-blank-duration being consistently lower than for the sine-wave fundamental.

The remaining two complex waveforms were identical in every respect except the phase relationship of their components. For the $180^{\circ}$ out of phase condition (waveform 5) critical-blank-durations were always greater than for the fundamental alone. while for the in-phase condition (waveform 4) critical-blankdurations were always lower.

This implies that blank elimination by complex waveforms is partly dependent on the phase relationship of their components; waveforms with components in-phase giving longer critical-blankdurations than similar waveforms with their components out of-phase. The relatively poor elimination by a square-wave could reflect the fact that all its harmonic components are in-phase.

\section{EXPERIMEYT 5}

\section{Effect of Phase on Elimmative Power of Complex Wateforms}

In these experiments the blank was alternated with a complex waveform made up of a "fundamental" sine-wave $(0.66 \mathrm{c} / \mathrm{deg})$ and its third harmonic (three times the frequency and one third the amplitude). The relative phase of these two components was varied between $0^{\circ}$ and $180^{\circ}$ at random between trials. For comparison, on some trials. the fundamental alone was presented in place of the complex waveform. Subjects made ten settings of critical-blank-duration at each of nine phase angles. and for the fundamental alone.

The subjects were not able to distinguish clearly between the nine different complex waveforms. but described all waveforms as falling into one of three groups:

(1) With phase differences of $60^{2}$ or less the appearance resembled the in-phase case.

(2) With phase differences of $120^{\circ}-180^{\circ}$ the appearance resembled the $180^{\circ}$ out-of-phase case.

(3) For intermediate phase differences the waveform alternated between appearing similar to the in-phase case, and similar to the $180^{\circ}$ out-ofphase case.

Figure 4 shows the varuation of critical-blank- 


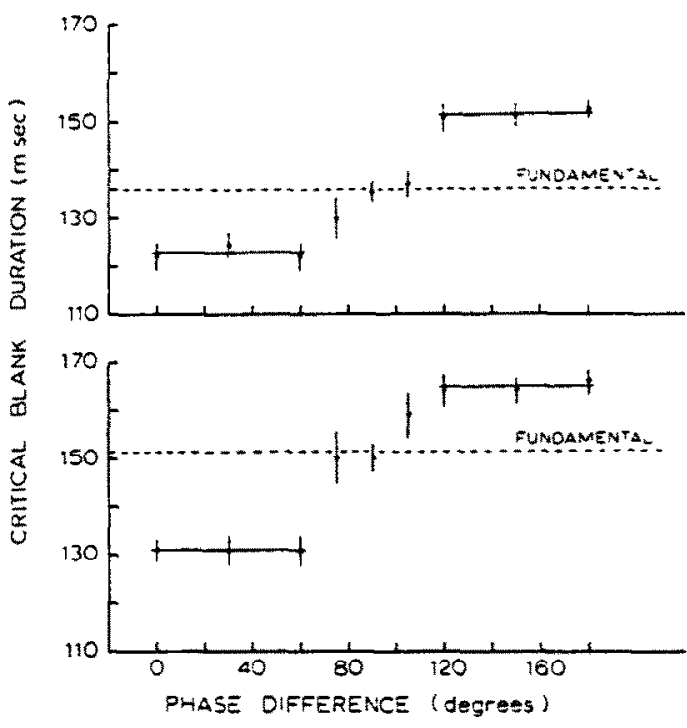

Fig. 4. Effect of phase on blank-elimination by complex waveforms. Upper graph, with filled circles. shows data for RC lower graph. with open circles. shows data for JPF. Horizontal broken line shows critical-blank-duration for the fundamental alone. Horizontal continuous lines represent the mean critical-blank-durations in plateau regions $\left(O=60^{\circ}\right)$ and $120^{\circ}-180^{\circ}$ phase difference. Vertical bars at each point represent \pm 2 S.E.

duration with phase. For phase differences of $120^{2}-180^{2}$ critical-blank-duration shows negligible variation and is longer than that for the fundamental alone. For phase difference of $60^{\circ}$ or less criticalblank-durations are shorter than that for the fundamental alone and also show negligible variation. For intermediate phase differences settings of criticalblank-duration are scattered between these upper and lower plateau regions.

The similarity in appearance between all waveforms of phase difference of $60^{\circ}$ or less. and that between all waveforms of phase difference $120^{\circ}-180^{\circ}$, could be linked with the closely similar critical-blank-duration settings within these two groups of complex waveforms shown in the data.

\section{Blank es Pattern Stimuli}

\section{EXPERIMENT 6}

We have now considered whether our failure to perceive an event of these considerable durations occurring on the retina is due to the unique nature of a grey-out. either produced by a saccade or artificially. as produced here. To test this we replaced the blank with either a grating of different frequency or other patterns such as a single bar. Again. mean luminance was kept constant.

Any other stimulus than a blank was found to be perceived dramatically well for exposures of $100 \mathrm{msec}$ or more. As the presentation time was decteased the apparent contrast of the inserted pattern naturally decreased due to the integration time of the visual system (Bloch's Law). When its duration was as low as 10 msec the pattern was still seen, but at low contrast. The CRT frame rate prevented testing shorter durations.

\section{DISCLSSION}

These experiments have shown that the perception of a blank. uniformly illuminated field can be eliminated for relatively long durations when preceded and followed by gratings. As stated. the use of the term "elimination" is not meant to imply any active inhibition of visual function by the gratings, but merely to refer to phenomenological failure to perceive the blank.

The eliminative power of a grating is highly dependent on its spatial frequency: the duration of blank which fails to be perceived increasing from about $80 \mathrm{msec}$ to $350 \mathrm{msec}$ for a rise in spatial frequency of 0.3 to $16 \mathrm{cideg}$. The parameter of contrast. however, has no effect at all on the eliminative power whatever the spatial frequency of the grating.

A square-wave eliminates the blank less effectuvely than does a sine-wave of the same fundamental frequency, despite the fact that it contains the higher odd harmonics. each of which alone would have more eliminative power than the fundamental in spite of their lower contrast.

Thus, it is clear that combining sine-waves does not result in an effect that is the simple algebraic addition of the eliminative properties of the separate components. Other factors affect the eliminative power of complex waveforms. notably the phase relationships of the components.

The lower power of a square-wave to eliminate perception of the blank becomes less surprising when the influence of phase is taken into account. The investigation of the elimination of perception by complex waveforms made up of two sine-wave com. ponents has shown that critical-blank-durations are significantly shorter when the components are inphase than when they are $180^{\circ}$ out-of-phase. A square-wave, all of whose components are in-phase. can therefore be expected to be of lower eliminative power than a sine-wave.

When the phase difference between the two components (fundamental and third harmonic) was varied between $0^{\circ}$ and $180^{\circ}$, the eliminative power of the complex waveform did not vary in a continuous manner. Instead. the visual system appears to be able to signal only two phase relationships $-0^{\circ}$ or $180^{\circ}$ phase difference. When components have $0^{*}-60^{*}$ phase difference the critical-blank-duration remains at a fixed level, while when the phase difference is $120^{\circ}-180^{\circ}$ it assumes a new, higher level. Between these two ranges the visual system seems insensitive to the specific phase relationship of the waveform components, and this is reflected in the intermediate nature of the eliminative properties. Thus the system seems to generalize for phase differences of $0^{2} \pm 60^{\circ}$ and of $180^{\circ} \pm 60^{\circ}$. categorizing them. respectively, as $0^{\circ}$ or $180^{\circ}$. Our observations support this hypothesis.

As stated. the subjective appearance of the complex waveform fluctuates with phase difference in a similar manner to its eliminative properties. This fluctuation was noted by Atkinson and Campbell (1974) during experiments on monocular rivalry, and later followed up by Furchner and Ginsburg (1976). They found that the degree of fluctuation in appearance in such waveforms as we have used was greatest when the two sine-wave components were between $60^{\circ}$ and $120^{\circ}$ 
out-of-phase, and assumed a much lower level when the components were $0^{\circ}-60^{\circ}$ or $120^{\circ}-180^{\circ}$ out-ofphase. Atkinson and Campbell (1974) attributed this to "interaction among spatial frequency selective channels whose components are stabilised by a phase selective device only for phase near $0^{\circ}$ or $180^{\circ \%}$. Our observations support this hypothesis.

\section{Apparent movement effect}

At blank durations equal to or shorter than the critical-blank-duration an apparent movement of the gratings was observed, as mentioned in the Results. It is important to note that the gratings preceding and following each blank were identical in spatial frequency and phase so that no real movement occurred.

When blank durations were well above the criticalblank-duration subjects reported the brief appearance of a negative afterimage immediately following the offset of the gratings (optically, a photographic negative of a grating is identical to the original positive except that it is shifted in phase by $180^{\circ}$ ). This resulted in the blank initially having the appearance of a low contrast sine-wave grating of the same spatial frequency as the preceding grating but advanced $180^{\circ}$ in phase with respect to it. This could give the overall appearance of a single grating shifting rapidly through 1 cycle on the screen.

However, this cannot be the complete explanation of the apparent movement since it was observed, particularly for high spatial frequency gratings, that the movement frequently appeared to be through more than 1 cycle. Another well-known effect which may contribute to this perceived motion is the Phi phenomenon-the apparent movement seen when two lights are alternately fiashed on and off some distance apart in the visual field. Although in our experimental situation the two gratings bracketing each blank period are identically placed in the visual field, due to their periodic nature it may be that non-equivalent bars in the two gratings are perceived as being the same bar shifted in space on temporally adjacent grating presentations. Thus a type of Phi movement of random amplitude and direction could occur.

\section{Blank elimination}

The brief presence of the negative afterimage of the grating might also explain the elimination either by effectively "filling-in" the blank or by causing it to be interpreted as a movement of the grating. In order to test this hypothesis a further experiment was carried out under similar conditions, but in which the formation of an afterimage was prevented.

The eliminating gratings, rather than being stationary, were phase-alternated by $180^{\circ}$ eight times per sec. This effectively prevented the formation of an afterimage because spatio-temporal changes in contrast produced by the grating would average out.

Settings of critical-blank-duration made by two subjects (JPF and RC) under these conditions were not significantly different from settings made when the gratings were stationary at the three spatial frequencies tested $(0.5,2.5$ and $10 \mathrm{c} / \mathrm{deg})$. Another way of ruling out the possibility of a significant contribution by afterimages was suggested and demonstrated by Edwin Land of the Polaroid Corporation. He viewed the test pattern through a slowly rotating
Dove prism so that the grating ortentation was continuously changing. This did not make the blank more visible despite the fact that there could be no afterimage. Therefore the elimunation is not dependent on the formation of a conventional afterimage of the eliminating grating. suggesting that the mechanism of elimination is not of photochemical origin (Sakitt. 1976).

\section{Visual masking}

There is a large body of literature on masking phenomena (see reviews by Kahnemann, 1968, and Raab, 1963), which relate to the interaction of stimuli presented close to one another in time and space. A visual masking explanation of the increased detection threshold for a flash during a saccade has already been proposed (Mackay, 1970: Matin. Clymer and Matin 1972). However. the effect described here cannot be interpreted in terms of "classical masking". the elimination of perception being specific to a blank. The use of the term "masking" has been avoided for this reason.

\section{Grey-out elimination and saccadic eye movements}

In experiments where some other feature, such as a second grating or a single bar. is used instead of a blank this is seen as a separate event down to durations as short as $8 \mathrm{msec}$. Thus. for events other than blanks, we always see what is actually happening in the visual world apart from the modifications inevitably imposed by the integration time of the visual system.

A brief blanking out of the visual world, that is, a sudden large decrement of contrast with little or no accompanying change in mean luminance, is never normally seen by the stationary eye. However. such a stimulus occurs every time the eye makes a saccade.

It is clear that a grey-out, whether it takes the form of the blank field discussed here or the saccadic smear referred to by Campbell and Wurtz (1978), is a unique visual stimulus the perception of which can be entirely eliminated when it is of sufficiently short duration and is preceded and followed by clear patterned images such as the gratings used here or the retinal images of fixations on a real scene, as used by Campbell and Wurtz (1978).

Our failure to see saccadic grey-out seems to be a built-in property of the visual system's response to a blank field. and not dependent on the actual movement of the eye.

It may be that a grey-out is regarded by the visual system as a kind of zero-input stimulus and that for the duration of such a stimulus the retinal message from the clear image preceding the grey-out is stored in the system and continues to be perceived. On the input of new pattern information the stored image is erased and the system is able to perceive the virgin image which follows the grey-out.

The data given here indicate that the system has a greater storage capacity for high spatial frequencies than for low, and that if maximum storage duration is indicated by critical-blank-duration then the former is also independent of the contrast of the stored image. 
Acknowledgements-Rachel Corfield is supported by a Medical Research Council grant. We wish to thank Clive Hood for his inestimable assistance. Denis Pelli for his help with electronics and computing. and Professor John Foley of the University of California. Santa Barbara. for acting as a subject.

\section{REFERENCES}

Atkinson J. and Campbell F. W. (1974) The effect of phase on the perception of compound gratings. Vision Res. 14. 159-162.

Campbell F. W. and Wurtz R. H. (1978) Saccadic omission: why we do not see a grey-out during a saccadic eye movement. This issue. pp. 1297-1303.
Furchner C. and Ginsburg A. P. (1976) Monocular rivalry" of a complex waveform: Phase and eye movement effects on pattern classification. ARVO 1976.

Kahnemann D. (1968) Method. findings and theory in studies of visual maskıng. Psychol. Bull. 70. 404-425.

Mackay D. (1970) Elevation of visual threshold by displacement of retinal image. Nature 225. 90-92.

Matin E.. Clymer A. B. and Matin L. (1972) Metacontrast and saccadic suppression. Science 178. 179-181.

Raab D. H. (1963) Backward masking. Psychol. Bull. 60. 118-129.

Sakitt B. (1976) Iconic memory. Psychol. Rev. 83. 257-276.

Stecher S.. Siegel C. and Lange R. V. (1973) Composite adaptation and spatial frequency interactions. Vision Res. 13, 2527-2531. 